\title{
Explanation of Resolution VIII proposed by the IAU/WGRS Sub-Group on Astronomical Constants
}

\author{
Toshio FUKUSHIMA \\ National Astronomical Observatory \\ 2-21-1, Obsawa, Mitaka, Tokyo 181, Japan \\ (Internet) toshio@spacetime.mtk.nao.ac.jp \\ (DECnet) UTSUN::" toshio@spacetime.mtk.nao.ac.jp" \\ (BITnet) toshio\%spacetime.mtk.nao.ac.jp@jpnkyoto \\ $(\mathrm{FAX})+81-422-41-3793$
}

\section{Background}

The IAU Working Group on Reference Systems (WGRS) Sub-Group on Astronomical Constants (SGAC) was established in June, 1989, as a consequence of resolutions adopted by Commissions 4 , 7,8 and 24 at the IAU General Assembly at Baltimore in 1988. The given missions of this sub-group were stated clearly by J. Hughes, the chairman of the WGRS, as:

\begin{abstract}
"Provide numerical values for the primary constants and specify the relationships between these and other, secondary constants within the framework of general relativity. This task will involve the documentation of the constants themseives as well as of the procedures and algorithms associated with their use. Recognition must be given to the fact that approaches which are specific to various techniques exist. The group must recommend the best estimates which can meet the varied requirements of astronomy. The apparent dichotomy between adopting fixed values for various quantities on the one hand, and the need for current, highly accurate values on the other hand, must be addressed by the group. Indeed, the crafting of effective procedures for incorporating new determinations into the values assigned to the constants, and the setting up of a mechanism for disseminating information regarding new determinations as an interim measure, are important tasks for this group."
\end{abstract}

\section{Discussions within the SGAC}

\subsection{Relativistic effects on units}

As stated in the paper of Fukushima et al. (1986, Celest. Mech. 36, 215), the IAU1976 convention on time-like arguments to have no secular difference among them forces one to use different sets of units in different coordinate systems within the framework of general relativity; the terrestrial meter and second in the geocentric coordinate system on the one hand, and the barycentric meter and second in the barycentric coordinate system on the other hand, for example. Furthermore both of these units differ from SI units. It was acknowledged that there are two options to solve this problem. The one is to keep 
the IAU1976 convention and to introduce different systems of units. In this case. a scaling factor should be introduced to connect different systems of units. The other is to abandon the IAU1976 convention and to use only one system of units, SI. The latter was chosen in compliance with other Resolutions. Yet it was noted that the numerical values of constants determined by using the TDB-based observation should be examined carefully. Thus, we judged that we could not prepare a self-consistent system of astronomical constants to the IAU General Assembly in 1991.

\subsection{Discrimination of constants and quantities}

The correspondences to the questionnaire indicated the tendency to extend the coverage of the system of astronomical constants to include, for example, the transformation matrix between FK5 and a galactic reference frame, the mean rotational angular velocity of the Earth $\omega, G M$ of many natural satellites and so on. However, it is clear that the degree of accuracy for estimated numerical values differs very much depending on the nature of the determination. Some constants have more than 10 welldetermined digits while others are estimated with $50 \%$ accuracy. Also it was argued that there are two contradictory requirements on constants; 1) to seek the latest and most accurate values and to update them as frequently as possible, and 2) to keep them as standards for long-term references. To solve this dilemma, It was proposed to discriminate primary and secondary constants and to call the latter as "best estimates". This proposal was welcomed by many members, however, to draw a line between them will require a deep consideration. Also many people felt that to extend the coverage was beyond the mission of the SGAC even if it was desirable.

\subsection{Need for standard procedures}

Almost all replies stressed the importance of providing standard procedures in fundamental astronomy. In other words, they required a kind of IAU version of the IERS Standards. The IERS Standards did not and will not cover the whole of fundamental astronomy so that another set of standards, say IAU Standards, is required. Also possible media for their distribution were discussed; to utilize E-mail systems or to provide them in a machine-readable form. However, some members argued that to prepare them was far beyond the mission of the SGAC. So it was proposed to establish a special working group for their establishment.

\subsection{Update mechanism}

The update mechanism of the system of astronomical constants being similar to that of the geodetic system of constants and their best estimates in the IAG was discussed and almost all members and consultants agreed to introduce a similar kind of mechanism into the IAU. Namely, to keep a system of constants for long-term references and to update a list of best estimates for other specified 'quantities' at every General Assembly.

\section{Resolution VIII}

Considering the above discussions and the fact that it was difficult to prepare a consistent system of astronomical constants this time, we proposed the following resolution (Resolution VIII);

The XXIst General Assembly of the IAU, 
recognizing,

a) the importance to astronomy of adopting conventional values of astronomical and physical constants,

b) that values of these constants should be unchanged unless they differ significantly from their latest estimates

c) that estimates of these constants should be improved frequently to represent the current status of knowledge,

d) the necessity of providing standard procedures using these numerical values, and, noting,

a) that the MERIT Standards and IERS Standards have contributed significantly to the progress of astronomy and geodesy,

b) that numerical values in these standards have served as a system of constants in analyzing obser. vations of high quality, and

considering,

that procedures in these standards do not cover the whole of fundamental astronomy, recommends,

that a permanent working group be organized by Commissions 4, 5, 8, 19, 24 and 31, in consultation with the IAG and the IERS, in order to update and improve the system of astronomical units ana constants, the list of estimates of fundamental astronomical quantities and standard procedures; this group shall:

1. prepare a draft report on the system of astronomical units and constants at least six months before the XXII General Assembly (1994),

2. prepare a draft list of best estimates of astronomical quantities at least six months before each following General Assembly,

3. prepare, at least six months before each following General Assembly, a draft report on standard procedures needed in fundamental astronomy, which,

a) should have a maximum degree of compatibility with the IERS Standards

b) should include the implementations of procedures in the form of software and/or test cases,

c) should be available not only in written form, but also in machine-readable form,

4. prepare a draft report on possible electronic access to these units, constants, quantities and procedures at least six months before the XXII General Assembly (1994). 
128

\section{Current Sets of Astronomical Constants}

Very recently, we, the SGAC, reported a set of best estimates of astronomical constants (Fukushima, 1991), which was augumented by the report of IAG/SSG/5.100 (Bursa. 1991). The combination of these two lists of latest estimates of astronomical/geodetic constants is named here temporally the SGAC1991 set, as an example of "list of best estimates of astronomical quantities" in the item 2 of the above resolution. Note that this set is neve: assured to be self-consistent!! The table below illustrates its differences from two sets of astronomical constants currently used as self-consistent systems; the IAU1976 system and IERS Standards (1989), where the latter we call here as the IERS1989 system. Note that the well-known planetary/lunar ephemerides DE200/LE200 has its own system of constants which is very similar to the IERS1989 system. Here the units for $k, G, G M_{E a r t h}, \omega$, and $G M_{S \text { un }}$ are $\mathrm{AU}^{3 / 2} /\left(\right.$ day $\left.^{1 / 2} M_{\text {Sun }}^{1 / 2}\right), 10^{-11} \mathrm{~m}^{3} /\left(\mathrm{kg} \mathrm{s}^{2}\right), 10^{14} \mathrm{~m}^{3} / \mathrm{s}^{2}, 10^{-5}$ radian $/ \mathrm{s}$, and $10^{20} \mathrm{~m}^{3} / \mathrm{s}^{2}$, respectively. In the table, a dagger denotes the defining constants, while the square brackets do the derived constants, and the dashes show that the corresponding item is not specified. The last one, the SGAC1991, will be a starting point for the next working group on astronomical standards (IAU/WGAS).

\begin{tabular}{llll}
\hline Constants (Unit) & IAU1976 & IERS1989 & SGAC1991 \\
\hline$k$ & $0.01720209895^{\dagger}$ & $0.01720209895^{\dagger}$ & $0.01720209895^{\dagger}$ \\
$c(\mathrm{~m} / \mathrm{s})$ & 299792458 & $299792458^{\dagger}$ & $299792458^{\dagger}$ \\
$\tau_{A}(\mathrm{~s})$ & 499.004782 & 499.00478370 & {$\left[499.0047835\left( \pm 210^{-7}\right)\right]$} \\
$\mathrm{AU}(\mathrm{km})$ & {$[149597870]$} & {$[149597870.66]$} & $149597870.61( \pm 0.05)$ \\
$G$ & 6.672 & 6.67259 & $6.6726( \pm 0.0003)$ \\
$a_{e}(\mathrm{~m})$ & 6378140 & 6378136 & $6378136.3( \pm 0.5)$ \\
$J_{2}(0.001)$ & 1.08263 & 1.082626 & $1.0826362\left( \pm 610^{-7}\right)$ \\
$G M_{\text {Earth }}$ & 3.986005 & 3.98600440 & $3.98600441\left( \pm 110^{-8}\right)$ \\
$\omega$ & - & - & $7.292115\left( \pm 110^{-6}\right)$ \\
$M_{\text {Moon }} / M_{\text {Earth }}(0.01)$ & 1.230002 & 1.2300034 & {$\left[1.2300034\left( \pm 1.510^{-6}\right)\right]$} \\
$p(" / \mathrm{jc})$ & 5029.0966 & 5029.0966 & $5028.85( \pm 0.06)$ \\
$\epsilon_{0}$ & $23^{\circ} 26^{\prime} 21^{\prime \prime} .448$ & $23^{\circ} 26^{\prime} 21^{\prime \prime} .4119$ & $23^{\circ} 26^{\prime} 21^{\prime \prime} .411\left( \pm 0^{\prime \prime} .002\right)$ \\
$M_{\text {Sun }} / M_{\text {Mercury }}$ & 6023600 & 6023600 & $6023600( \pm 250)$ \\
$M_{\text {Sun }} / M_{\text {Venus }}$ & 408523.5 & 408523.5 & $408523.71( \pm 0.06)$ \\
$M_{\text {Sun }} /\left(M_{\text {Earth }}+M_{M \text { oan }}\right)$ & {$[328900.5]$} & {$[328900.55]$} & $328900.55( \pm 0.01)$ \\
$M_{\text {Sun }} / M_{\text {Mars }}$ & 3098710 & 3098710 & $3098708( \pm 9)$ \\
$M_{\text {Sun }} / M_{\text {Jupiter }}$ & 1047.355 & 1047.350 & $1047.3486( \pm 0.0008)$ \\
$M_{\text {Sun }} / M_{\text {Saturn }}$ & 3498.5 & 3498.0 & $3497.90( \pm 0.02)$ \\
$M_{\text {Sun }} / M_{\text {Uranus }}$ & 22869 & 22960 & $22902.94( \pm 0.04)$ \\
$M_{\text {Sun }} / M_{\text {Neptune }}$ & 19314 & 19314 & $19412.24( \pm 0.06)$ \\
$M_{\text {Sun }} /\left(M_{\text {Pluto }}+M_{\text {Charon }}\right)$ & $310^{\circ}$ & $1.310^{8}$ & $1.35( \pm 0.05) 10^{8}$ \\
\hline
\end{tabular}

IAU1976 Duncombe et al.: 1977, Transactions of the IAU, XVIB, 56.

IERS1989 McCarthy et al.: 1989, IERS Tech. Note, No.3, 1.

SGAC1991 Fukushima: 1991, Proc. of IAU Coll. No.127, 27.

Bursa: 1991, Report of $I A G / S S G / 5.100$, to be presented at the IUGG General Assembly in 1991. 


\section{DISCUSSION}

Vicente:

I have a few comments on your remarks. The first is about the consistency between the IAU and the IUGG. Since 1980 we have had a consistent system. Since 1976 there was a working group of the IAU and a working group of the IAG and we did publish, the International Association of Geodesy did publish a consistent system. I was a member of that working group. So since those days things have been fairly consistent. Second question; you spoke about precession difficulties. You mention that lunar laser ranging has already oue revolution, but you must remember lunar laser ranging has very few stations and we know now-a-days that Earth is a very complicated planet. Don't forget plate tectonics. So that is a very important point. Another one I should like to mention about your Recommendation, and I like it very much that you say here in " $b$ ", that values of this constant should be unchanged, unless they deviate significantly from their latest estimates. I find your Recommendation VIII and Recommendation $V$, which means that you should keep the old time systems, are really not very consistent with Recommendations I through IV. That's one thing I should like to point out. Another one is about the future working group, I hope that the future working group represents the views of different people and is not very one-sided, that only people having the same ideas are in that working group. That's my best wish.

\section{Fukushima:}

As for the relation with the IAG and IUGG people, I direct your attention to the constitution of this new permanent working group. There is a phrase, "in consultation with the IAG and the IERS," and I feel that this phrase should cover these things. Inviting a member from IAG as a representative should avoid differences between the geodesy people and the astronomical people. Anyway I am sure to keep it in mind. And as for the lunar laser ranging, your remarks are quite correct. We should have some work at several other Observatories. 